\title{
Immunohistochemical evaluation of angiogenesis in warts
}

\author{
Simon Bossart*, Lisa Hartmeier, S. Morteza Seyed Jafari, Helmut Beltraminelli and Robert E. Hunger \\ Department of Dermatology, Inselspital, Bern University Hospital, University of Bern, Switzerland
}

\begin{abstract}
Objectives: Angiogenesis is a key event in tumour growth. An infection with human papillomavirus (HPV) typically leads to benign tumours, such as warts (papillomas) on the skin and mucous membranes. The role of angiogenesis in skin warts is yet to be established.

Materials and methods: The present work evaluates the hypothesis that the development of skin warts is associated with alterations in the dermal vascularisation (dermal microvessel density/mm², MVD). Seventeen cases of cutaneous warts and eight cases of normal skin were studied. Dermal vessels were immunostained for CD31, CD34 and CD105. The ten areas with the highest number of microvessels were counted.

Results: The number of CD31+ ${ }^{+}$CD $34^{+}$and CD105+ vessels was significantly higher in cutaneous warts compared to that in normal skin (mean \pm SD: $45.71 \pm 22.63$ vs $21.27 \pm 5.83, \mathrm{P}=0.0062 ; 59.75 \pm 24.93$ vs $17.13 \pm 5.67, \mathrm{P}<0.001 ;$ and $29.74 \pm 7.09$ vs $6.58 \pm 1.89, \mathrm{P}<0.001$ respectively).

Conclusions: Higher MVD values in skin warts suggest a possible causal correlation between angiogenesis and development of papillomas in HPV infected skin. Our results shows the morphological histopathological explanation (high number of vessels) for the punk-shaped vessels seen in the dermatoscopical examination.
\end{abstract}

\section{Introduction}

Skin warts are benign tumours caused by an Human Papilloma Virus (HPV) infection of the keratinocytes. HPV is a DNA virus belonging to the papillomavirus family which contains over 200 genetically distinct strains. It infects the epithelia or the mucosa of the skin and mostly causes benign papillomas or skin warts [1]. Moreover, certain oncogenic HPV types $16,18,31,33,45,52$, and 58 may cause anogenital, oropharyngeal, and skin cancers, such as squamous cell carcinoma [2-3].

Although it is known that HPV plays a major role in the development of warts, the exact pathophysiological mechanism of tumour growth is still unclear. In all HPV infections, newly formed dilated blood vessels are present. In cutaneous warts, these vascular structures may be seen with the dermatoscope in form of multiple papillae containing a central red or punk-shaped black dot or loop surrounded by a whitish halo, also described as thrombosed capillaries [4]. Therefore angiogenesis and vasodilatation are considered to be involved in the development of warts [4-6]. Angiogenesis can be quantified by assessing the microvessel density (MVD), which reflects the number of vessels per $\mathrm{mm}^{2}$ within a histological section, and is associated with the degree of local neovascularisation [7]. Immunohistochemical endothelial markers such as CD31, CD34 and CD105 are used to identify endothelial cells and to detect local vascularisation $[8,9]$.

CD31 (Platelet endothelial cell adhesion molecule, PECAM-1) is a single chain type- 1 transmembrane protein that plays a role in endothelial cell-cell junctions, as well as leukocyte-endothelial cell junctions [10]. CD34 is also a single-chain transmembrane glycoprotein that is present in haematopoietic precursor cells and endothelial cells of blood and lymphatic vessels [11].

Endoglin (CD105) is an accessory receptor for transforming growth factor-beta (TGF- $\beta$ ), and its expression is up-regulated in actively proliferating endothelial cells. Endoglin is an appropriate marker for angiogenesis and neovascularisation [12-13].
To date, the exact role of angiogenesis in skin warts has not been established. Therefore, the present work aimed to evaluate the immunohistochemical expression of CD31, CD34 and CD105 in common skin warts. We assume that these markers could be related to papilloma-dependent neoangiogenesis in HPV infected cells.

\section{Main text}

\section{Methods}

Study population: Seventeen biopsies of histologically and clinically confirmed skin warts and eight biopsies of normal skin were included in the study to compare neoangiogenesis. The Inclusion criteria included samples of skin warts excised from the hands or feet of the patients who had not received any local medical treatment before the study. The medical history of all 25 patients was reviewed retrospectively focusing on age, sex, immune system status, and immunosuppressive medication use. The cantonal ethics commission of Bern approved the data analysis (KEK number 2018-00174).

Immunostaining and evaluation of CD31, CD34 and CD105: In order to compare the amount of dermal microvessels, immunohistochemical staining were performed using anti-CD31 (Dako IS610), anti-CD34 (Leica PA0212) and anti-CD105 antibodies (MA5-11854). The stained samples were scanned using a Pannoramic MIDI slide scanner (3DHISTECH). Acquired images were analysed using Pannoramic Viewer 1.15.4 (3DHISTECH). In each section, a total

${ }^{*}$ Correspondence to: Simon Bossart, MD, Inselspital Bern Universitatsklinik fur Dermatologie University Hospital, Inselspital Bern, 3010 Bern, Switzerland, Tel: +41 3163208 81; Fax: +41 3163222 33; E-Mail: simon.bossart@insel.ch

Key words: human papillomavirus, angiogenesis, warts, immunohistochemistry, punk-shaped vessels

Received: September 22, 2020; Accepted: September 28, 2020; Published: October 01, 2020 
of 10 different fields with the highest number of microvessels in one flap section at 200x magnification were selected and the vessel number was independently counted by two staff members. Vascularity was quantified in the stroma close to the epithelium up to approximately $500 \mu \mathrm{m}$ from the basal cell layer. The vessel density was calculated as the number of vessels per square millimetre field, and the mean was reported for two groups.

\section{Statistical analysis}

All analyses were conducted using the GraphPad Prism version 8.01 (GraphPad Software, Inc. USA). Descriptive statistics were presented for the animals in mean $\pm \mathrm{SD}$. All p-values represent two-sided tests with an alpha level of 0.05 . Mann Whitney test was used to detect the differences between groups.

\section{Results}

Twenty five samples were used in this study, including seventeen skin warts and eight control samples. The mean age of the patients was 52.91, and no significant difference was observed between the warts and control group with respect to age and sex. Nine patients (53\%) in the warts group had undergone immunosuppressive therapy after organ transplantation.

The immunostaining assay showed that $\mathrm{CD} 31+, \mathrm{CD} 34+$ and CD105+ vessel density was significantly higher in skin warts compared to that in normal skin, where the statistical data were represented as mean $\pm \mathrm{SD}(45.71 \pm 22.63$ vs. $21.27 \pm 5.83, \mathrm{p}=0.0062 ; 59.75 \pm 24.93$ vs. $17.13 \pm 5.67, \mathrm{p}<0.001 ; 29.74 \pm 7.09$ vs. $6.58 \pm 1.89, \mathrm{p}<0.001$; for CD 31 and CD 34, CD105 respectively; (Figures 1 and 2).

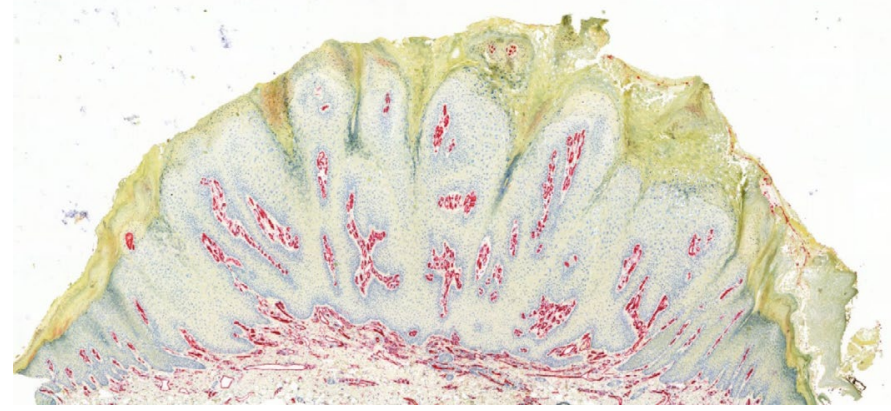

Figure 1A. CD $31^{+}$vessels in the upper dermis (stained red) in a wart sample $(\times 10$ original magnification)

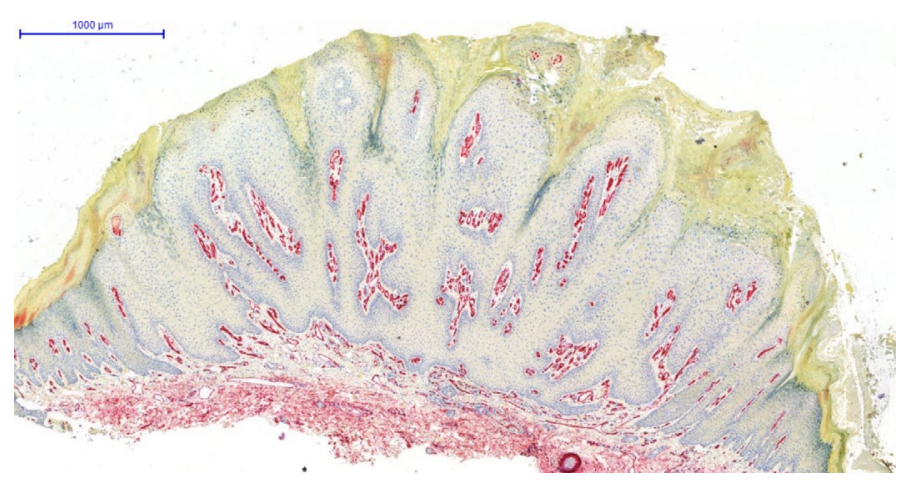

Figure 1B. CD $34^{+}$vessels in the upper dermis (stained red) in a wart sample $(\times 10$ original magnification)

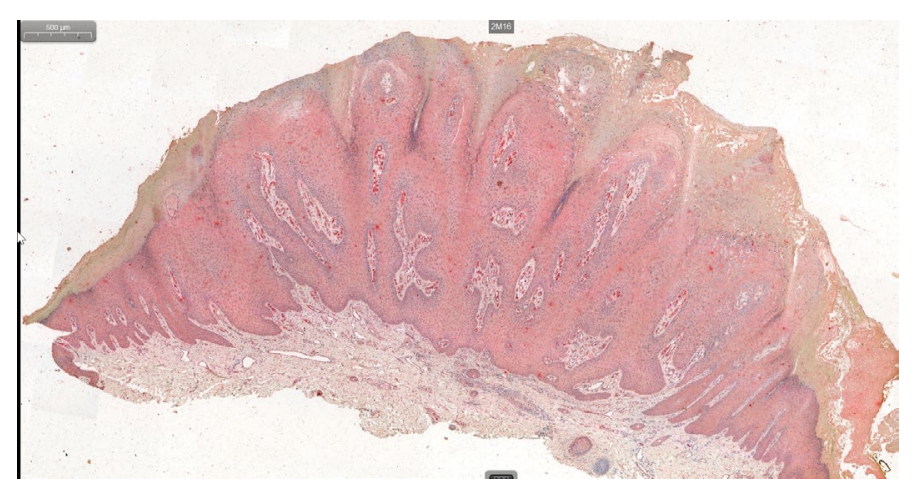

Figure 1C. CD $105^{+}$vessels in the upper dermis (stained red) in a wart sample $(\times 10$ original magnification)

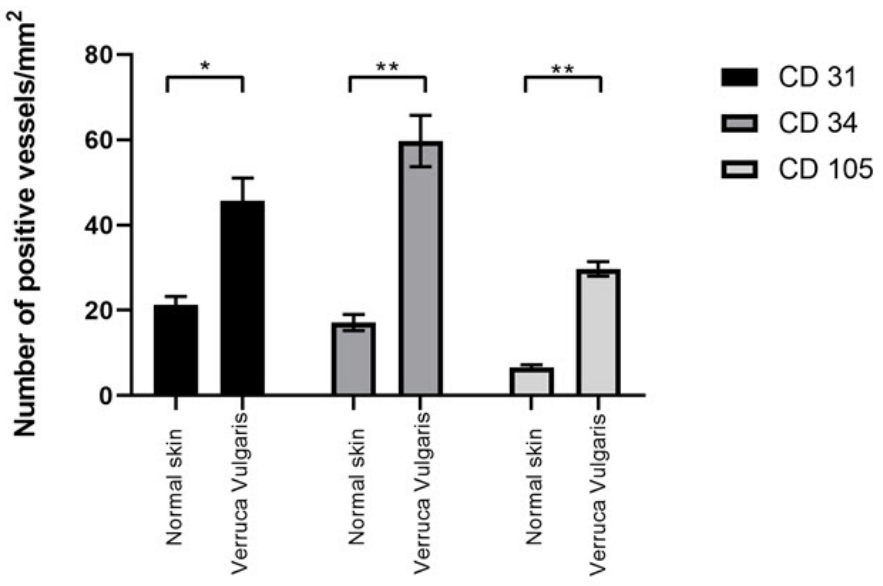

Figure 2. Significantly higher $\mathrm{CD} 31^{+}, \mathrm{CD} 34^{+}$and $\mathrm{CD} 105^{+}$vessel density in skin warts compared to the normal skin. $(* \mathrm{P}-$ Value $=0.0062, * *$ P-Value $<0.0001)$

\section{Discussion}

Angiogenesis plays a key role in primary tumour growth and can be quantified by the evaluation of the microvessel density, which reflects the number of vessels per $\mathrm{mm}^{2}$ within a histological section [6]. Vascular structures, spotted by dermatoscopy, are a characteristic clinical sign for the diagnosis of a skin wart [3]. However, the exact causative association of neovascularisation and the development of cutaneous warts remains unknown. CD31, CD34 are used as vascular endothelial markers, CD105 is helpful to assess neoangiogenesis. CD31 and CD34 are expressed in numerous tumour vessels, but they are also positive in both normal vessels and activated vessels, whereas CD105 has a higher affinity to activated endothelial cells [14-15]. Several previous studies have shown that $\mathrm{CD} 105^{+}$vessels are newly formed and are fragile, they can easily tear and bleed [15-16].

Our results indicate that a higher MVD in skin warts suggests a possible link between angiogenesis and the development of papillomas in HPV-infected skin. Moreover, the presence of CD $105^{+}$vessels could be the reason for the punk-shaped vessels visible dermatoscopically, which are also described by other authors as thrombosed capillary loops [4].

\section{Conclusions}

To our knowledge, this work is the first attempt to investigate the immunohistochemical detection of microvessels in skin warts. 
Our results provide evidence of angiogenetic activity by using CD31, CD34 and CD105 immunohistochemistry in skin warts, and indicate that local microvasculature may undergo papilloma-dependent angiogenesis. Further research is needed to investigate the possible causative role of angiogenetic factors in the development of skin warts as well as their potential benefit as therapeutic targets in the treatment of this skin infections.

\section{Limitations}

However, our study has several limitations. The data were analysed retrospectively, and the study population was rather small. In evaluating immunohistochemical results, there are possible causes for poor staining results, which can be unwanted background staining, artifactual staining or weak or absent staining.

\section{Declarations}

\section{Ethics approval and consent to participate}

The study protocol was approved by the cantonal ethic commission of Bern, Switzerland (KEK number 2018-00174). Informed written consent was obtained from all individual participants included in the study.

\section{Consent to publish}

Not applicable.

\section{Availability of data and materials}

All data generated or analyzed during this study are included in this published article.

\section{Competing interests}

The authors have no conflicts of interest to declare.

\section{Funding}

The authors received no financial support for the research, authorship, and/or publication of this article.

\section{Author Contributions}

All authors participated in analysing the data on which the conclusions and results are based, and writing the paper. SB, MJ and HB designed the study. SB, LH and MJ gathered and generated the data. All authors have read and approved the manuscript.

\section{Acknowledgment}

Thanks to Ursula Läderach for the valuable support in the laboratory.

This manuscript has been released as a pre-print at Research Square [17].

\section{References}

1. de Villiers EM (2013) Cross-roads in the classification of papillomaviruses. Virology 445: 2-10. [Crossref]

2. Fuller C, Hudgins E, Finelt N (2017) Human-papillomavirus-related disease in pediatrics. Curr Opin Pediatr 14: 1097. [Crossref]

3. Zur Hausen H. Papillomavirus infections - a major cause of human cancers. Biochim Biophys acta 1288: F55-F78. [Crossref]

4. Marghoob AA, Malvehy J, Braun RP (2012) An Atlas of Dermoscopy. (2 ${ }^{\text {nd }}$ Edn) CRC Press.

5. Harada K, Baillie R, Lu S, Syrjänen S, Schor AM (2001) VEGF expression in skin warts. Relevance to angiogenesis and vasodilation. Arch Dermatol Res 293: 233-238. [Crossref]

6. El-Nabarawy EA, El-Hanafy GM, Rashed LA, Yasin FS (2016) Expression of angiopoietin-1, angiopoietin-2, and their receptor Tie2 in verruca vulgaris (common skin warts). Int J Dermatol 55: e327-e31.7. [Crossref]

7. Folkman J (2003) Fundamental concepts of the angiogenic process. Curr Mol Med 3: 643-651. [Crossref]

8. Müller AM, Hermanns MI, Skrzynski C, Nesslinger M, Müller KM, et al. (2002) Expression of the endothelial markers PECAM-1, vWf, and CD34 in vivo and in vitro. Exp Mol Pathol 72: 221-229. [Crossref]

9. Fonsatti E, Nicolay HJ, Altomonte M, Covre A, Maio M (2010) Targeting cancer vasculature via endoglin/CD105: A novel antibody-based diagnostic and therapeutic strategy in solid tumours. Cardiovasc Res 86: 12-19.

10. Parums DV, Cordel JL, Micklem K, Heryet AR, Gatter KC, et al. (1990) JC70: A new monoclonal antibody that detects vascular endothelium associated antigen on routinely processed tissue sections. J Clin Pathol 43: 752-757. [Crossref]

11. Fina L, Molgaard HV, Robertson D, Bradley NJ, Monaghan P, et al. (1990) Expression of the CD34 gene in vascular endothelial cells. Blood 75: 2417-2426. [Crossref]

12. Burrows FJ, Derbyshire EJ, Tazzari PL, Amlot P, Gazdar AF, et al. (1995) Upregulation of endoglin on vascular endothelial cells in human solid tumors: implications for diagnosis and therapy. Clin Cancer Res 1: 1623-1634, [Crossref]

13. Kumar S, Ghellal A, Li C, Byrne G, Haboubi N, et al. (1999) Breast carcinoma: vascular density determined using CD105 antibody correlates with tumor prognosis. Cancer Res 59: 856-861. [Crossref]

14. Tanaka F, Otake Y, Yanagihara K, Kawano Y, Miyahara R, et al. (2001) Evaluation of angiogenesis in non-small cell lung cancer: Comparison between anti-CD34 antibody and anti-CD105 antibody. Clin Cancer Res 7: 3410-3415. [Crossref]

15. Ryu Fukumitsu, Yasushi Takagi, Kazumichi Yoshida, Susumu Miyamoto (2013) Endoglin (CD105) is a more appropriate marker than CD31 for detecting microvessels in carotid artery plaques. Surg Neurol Int 4: 132. [Crossref]

16. Mofidi R, Crotty TB, McCarthy P, Sheehan SJ, Mehigan D, et al. (2001) Association between plaque instability, angiogenesis and symptomatic carotid occlusive disease. $\mathrm{Br}$ J Surg 88: 945-950. [Crossref]

17. Bossart S, Hartmeier L, Seyed Jafari SM, Beltraminelli H, Hunger RE (2020) Immunohistochemical Evaluation of Angiogenesis in Warts. Research Square pp: 1-8.

Copyright: $\odot 2020$ Bossart B. This is an open-access article distributed under the terms of the Creative Commons Attribution License, which permits unrestricted use, distribution, and reproduction in any medium, provided the original author and source are credited. 